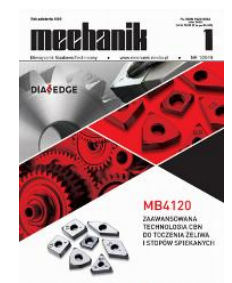

How to cite this article:

Authors: Marcin Januszka, Rafał Napierała

Title of article: „Application of virtual and augmented reality techniques for visualizing the results of FEM numerical analyses"

Mechanik, No. 1 (2019)

DOI: https://doi.org/10.17814/mechanik.2019.1.13

\title{
Application of virtual and augmented reality techniques for visualizing the results of FEM numerical analyses
}

\author{
MARCIN JANUSZKA \\ RAFAE NAPIERAEA* \\ Dr inż. Marcin Januszka, marcin.januszka@polsl.pl, https://orcid.org/0000-0002-0391-8023 - Politechnika Śląska, Wielton S.A., Gliwice, \\ Polska \\ Mgr inż. Rafał Napierała, rafal.napierala@polsl.pl, https://orcid.org/0000-0001-7595-7796 - Politechnika Śląska, Wielton S.A,. Gliwice, \\ Polska
}

An innovative approach for presenting the results of numerical FEM analysis is presented. Proposed visualization method uses virtual and augmented reality techniques for this purpose. The practical application of the new tool is presented on the example of strength analysis of the side beam of the curtainsider semi-trailer.

KEYWORDS: augmented reality, virtual reality, FEM analysis, visualization

\section{Introduction}

Numerical analyses have long been the basic tool for verification of developed products. They allow to detect the flaws of a new technical measure at the design stage, which significantly reduces the cost and time of preparing the final product in accordance with the adopted criteria.

The need to ensure an appropriate degree of strength and durability as well as weight reduction are the main reasons for the increase in the complexity of the structure, and thus also the verification process. It is not surprising that the correct interpretation of the results of numerical analyzes is becoming more and more difficult, especially in the case of complex systems. It requires from the researcher not only in-depth substantive knowledge, but also the skills of spatial reasoning.

Viewing the results of the analysis in the form of a graphic texture applied to the 3D model of the examined object on a flat screen monitor or projector does not always facilitate the interpretation of the results. Only longer manipulation of the displayed image in space allows you to see important details.

The dynamic development of computer techniques in recent years has created an effective alternative in the field of 3D objects presentation. The technique of so-called augmented reality (AR) enables the transfer of virtual objects to the surrounding reality and thus creating a uniform work environment [1]. If, for some reason, access to the examined object is difficult (environment harmful to humans, the physical object has not yet been created or is located within a distance of several hundred kilometers), virtual reality (VR) techniques come to the aid [2]. It allows to carry out the entire stage of presenting analysis results in a virtual environment.

The paper presents AR techniques and - in some common areas - VR as modern visualization tools used to present the results of FEM numerical analysis. The implementation of both techniques in the design process is to reduce the time needed to verify the results of numerical analyses and to facilitate the modification of the tested technical means.

The tests used the results of the strength analysis carried out as part of the development work carried out at Wielton in cooperation with the Silesian University of Technology. The subject of the research was verification of the state of stress in the so-called SL (safety lock) slat of the semi-trailer, to which cargo straps are attached to protect the transported goods. 


\section{System structure}

The basic tool, on which the system developed by the authors is based, is Vuforia Studio by PTC [3]. It is a web application for creating an AR system scene and for subsequent project management. Developing the stage is intuitive and does not require specialized knowledge in the field of programming and image processing, and at the same time allows you to prepare professional applications of augmented reality. The Vuforia Studio environment is complemented by the PTC Vuforia View application, which allows visualization of a previously prepared virtual scene using a tablet, smartphone or HMD display.

Developed system is based on the HMD Microsoft HoloLens display, which provides high quality visualization. The last tool needed to build the entire system is the CAD/CAE environment, which is used to develop 3D models and perform FEM analyzes used in the AR system.

The process of creating an AR application consists of several stages: preparation of 3D models, conducting a FEM numerical strength analysis, saving the results in a data repository, making an AR scene and its presentation. The first three steps can be implemented in any CAD program.

First, one needs to model the elements / subassemblies for use in the scene, i.e. in the virtual space that will be superimposed on the real image [4]. The Vuforia Studio environment, in which all 3D objects are loaded at the next stage, allows you to work with files in many popular formats (e.g.: .igs, .iges, .obj, .prt, .stp, .stl, .ipt, iam) [5]. The analysis results to be presented should also be prepared as a 3D object with the results texture applied.

The example shown uses the .wrl file format created in Creo Simulate 3.0 [6].

The second step in creating the application is the implementation of prepared elements in the AR scene. This stage boils down to loading models and choosing the method of orientation of the scene in real space (using a marker, by indicating a flat surface or object), as well as choosing the type of target display device - either it is a tablet, smartphone or HMD MS HoloLens display.

Objects inserted into the scene can be freely moved, rotated and resized. However, more advanced editing of these objects is not available as part of the AR scene; this can only be done in the CAD/CAE environment. However, it is possible to add 2D images and predefined markers. The finished scene is saved in a global database.

The last step is to display the project. The whole process is carried out using the Vuforia View application installed on the display device. After logging in to the account (on this device), access to previously created projects in the Vuforia Studio environment is gained. One needs to select a specific scene and display it after correct orientation in the real space.

The structure of the discussed system is shown in fig. 1.

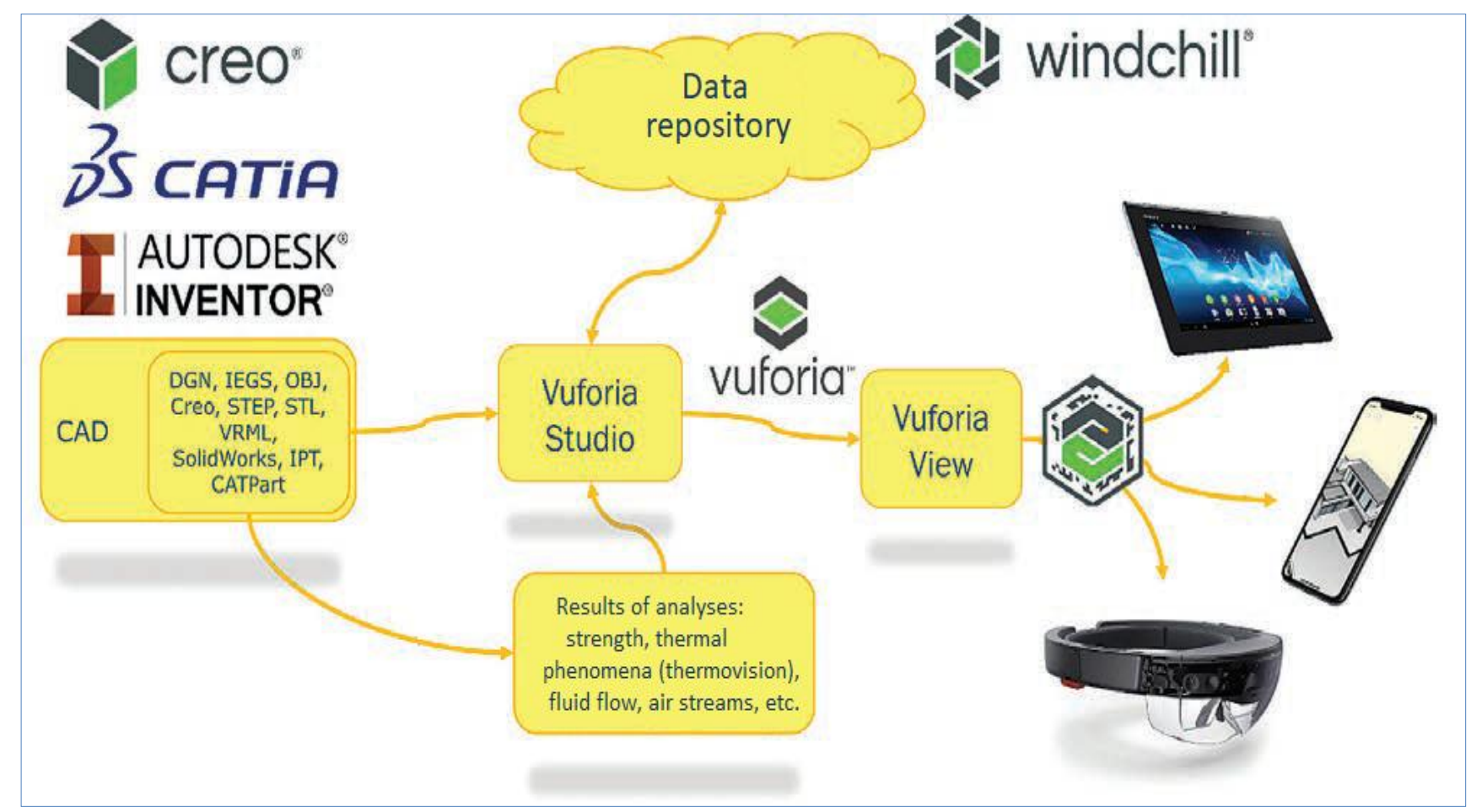

Fig. 1. AR/VR system structure 


\section{Application of AR techniques}

The first test of the AR technique within the framework developed by the authors of the environment took place inside the building, in a closed room. The 3D model with the results of the analysis was displayed on the table top next to the lying real product. The quality of the visualization was very good.

Virtual model had a high level of sharpness and contrast regardless of the perspective from which it was viewed. It was easy to change its size and location, which translated into quick adaptation to the real product (in this case a semi-trailer).

The augmented reality technique works great for quick analysis of results (fig. 2). In this effective way, the project team can jointly interpret them. Each team member, if they have the appropriate equipment in the form of a head-mounted display, sees the virtual model with FEM results from their own perspective, displayed directly on the table.

The second test consisted of displaying 3D results on the real element of the semi-trailer under the open sky. Quality of the virtual object was also good in this case. However, it should be noted that high sunlight deteriorates the graphic quality of the object. Sensitivity to weather conditions is the only factor causing some inconvenience. The location of the virtual model in the external environment (outside buildings) is carried out correctly. Additional displayable markers, in the form of given force vectors, were clearly visible against the background of other trailer components. The effect of visualization outside buildings using a real object is shown in fig. 3.

Validation studies of the developed system have shown that the use of augmented reality technology is possible in both office and field conditions. The use of such presentation methods is supported by high mobility and lack of attachment to a display device such as a monitor or projector. Due to the use of HMD (head-mounted display) displays, the analysis results can be shown on any surface. Access to prepared simulations is also problem-free, and all files needed to visualize the results are stored on the cloud server. Any authorized person may use them freely. No restrictions on the size of the subject of research is another advantage of the techniques discussed.

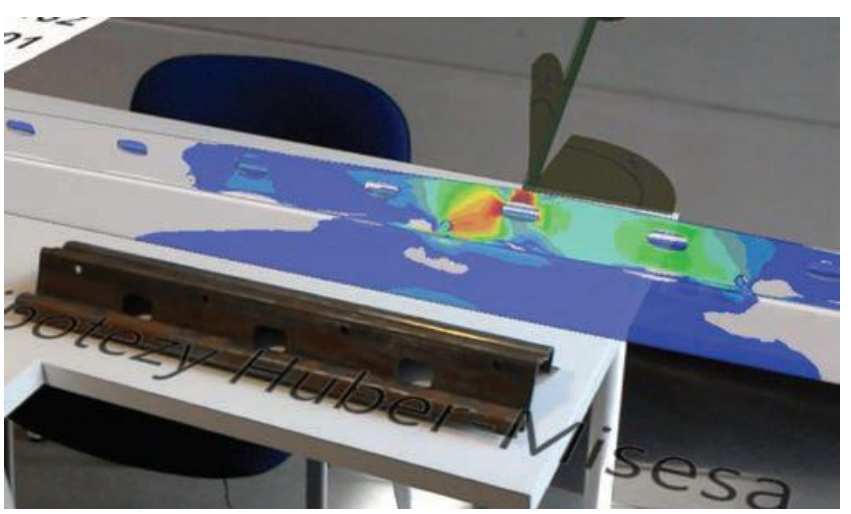

Fig. 2. Projection of the virtual 3D model with superimposed texture of the results of FEM analysis

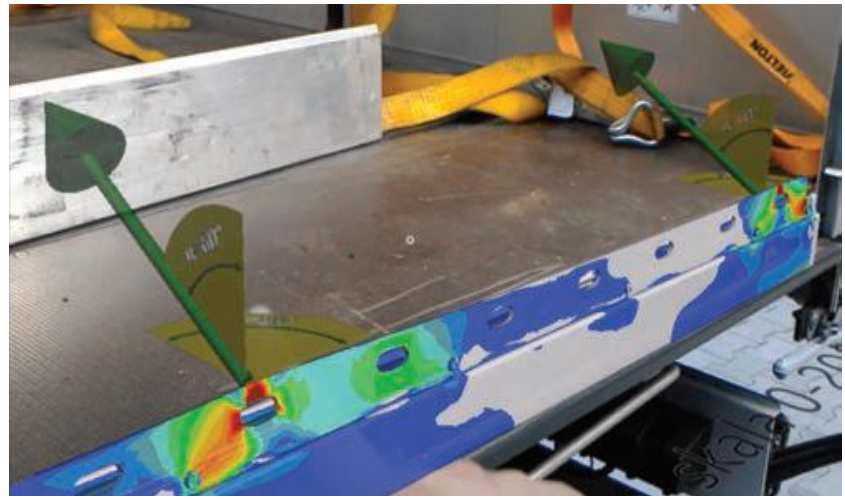

Fig. 3. Visualization of virtual FEM analysis results on a real research subject

Developed system allows the presentation of the results of analyses of the entire model at any scale, also 1:1. An example would be the SL strip, approximately $1.5 \mathrm{~m}$ long, shown in fig. 2 and fig. 3, which was displayed on a 1:1 scale and was analyzed in this form.

Visualized results of FEM analysis can also be enriched with additional elements, e.g. determining boundary conditions, such as values and directions of forces, or the adopted method of support in the case of strength analyzes. The result of using AR techniques at the stage of verification of the results of numerical strength analysis is a much faster detection of structural defects. Thanks to the presentation of FEM simulation results directly on a real object (in this case a semi-trailer), you can naturally see the elements and sub-assemblies that - when located near a critical area - can affect the phenomena occurring there. This definitely facilitates the interpretation of the results obtained and presented. On the monitor screen, where only a small part of the object is visible, it is more difficult to find these dependencies.

The use of AR techniques also ensures the comfort of analysis carried out by a group of people. With HMD displays or a regular smartphone at their disposal, each of them can view the area of interest regardless of the others (from their own perspective). Simultaneous analysis of the results of various fragments of the research object results in a direct reduction of the total verification time of the entire object. This is another advantage of modern AR presentation techniques. 
In addition to some dependence of image quality on sunlight, an additional disadvantage of these techniques are costs. The value of one professional HMD display is comparable to the price of about 15 monitors. However, the value of one software license (for commercial use) for building the AR scene can be compared with the price of seven CAD system licenses.

These costs can be an obstacle to the dissemination of new techniques, especially for small businesses. In the future, along with the popularization of software and hardware components, it is expected that costs will decrease, and tools of this type will be available not only to large enterprises with large R\&D centers.

\section{Conclusions}

The use of augmented reality techniques to visualize the results of analyzes creates new presentation possibilities. It enables comfortable viewing of the research object regardless of its working environment, size or number of people participating in the presentation. It facilitates correct interpretation and reduces the time needed to develop a modification of the structure in the event of irregularities.

Presented system is universal. The wide list of supported formats facilitates its use for many other types of numerical analyzes regarding such issues as thermal phenomena, fluid flow or air stream. It is expected that as technology develops, the cost of hardware and necessary software will decrease, and AR techniques will be increasingly used in industry and on a daily basis.

Research financed from the funds of the Ministry of Science and Higher Education on the basis of contract No. 12/DW/2017/01/1 of 07.11.2017 in the amount of PLN 4433520.00.

\section{REFERENCES}

[1] Azuma R.T. "A survey of augmented reality". Teleoperators and Virtual Environments. 6, 4 (1997): 355-385 .

[2] Billinghurst M., Clark A., Lee G. "A survey of augmented reality". Foundations and Trends in HumanComputer Interaction. 8, 2-3 (2014): 73-272.

[3] Vuforia Studio PTC, https://www.ptc.com/en/products/augmented-reality/vuforia-studio (access: 29.11.2018).

[4] Januszka M., Krysta W. „Projektowanie ergonomiczne z zastosowaniem technik poszerzonej rzeczywistości”. Mechanik. 91, 1 (2018): 82-84, https://doi.org/10.17814/mechanik.2018.1.20.

[5] Wspierane formaty plików wsadowych, https://support.ptc.com/help/vuforia/studio/en/\#page/Studio_Help_Center\%2FSupportedCADFileFormats.html (access: 29.11.2018)

[6] Creo Simulate PTC, https://www.ptc.com/en/products/cad/creo/simulate (access: 29.11.2018). 